\title{
Photoelectron balance in the dayside Martian upper atmosphere
}

\author{
XiaoShu Wu1,3, Jun Cui'2,3* Jiang Yu4 ${ }^{4}$ LiJuan Liu'2,3, and ZhenJun Zhou ${ }^{2,3}$ \\ ${ }^{1}$ National Astronomical Observatories, Chinese Academy of Sciences, Beijing 100101, China; \\ ${ }^{2}$ School of Atmospheric Sciences, Sun Yat-sen University, Zhuhai Guangdong 519082, China; \\ ${ }^{3}$ Chinese Academy of Sciences Center for Excellence in Comparative Planetology, Hefei 230026, China; \\ ${ }^{4}$ Space Science Institute, Macau University of Science and Technology, Macau, China
}

\begin{abstract}
Photoelectrons are produced by solar Extreme Ultraviolet radiation and contribute significantly to the local ionization and heat balances in planetary upper atmospheres. When the effect of transport is negligible, the photoelectron energy distribution is controlled by a balance between local production and loss, a condition usually referred to as local energy degradation. In this study, we examine such a condition for photoelectrons near Mars, with the aid of a multi-instrument Mars Atmosphere and Volatile Evolution data set gathered over the inbound portions of a representative dayside MAVEN orbit. Various photoelectron production and loss processes considered here include primary and secondary ionization, inelastic collisions with atmospheric neutrals associated with both excitation and ionization, as well as Coulomb collisions with ionospheric thermal electrons. Our calculations indicate that photoelectron production occurs mainly via primary ionization and degradation from higher energy states during inelastic collisions; photoelectron loss appears to occur almost exclusively via degradation towards lower energy states via inelastic collisions above $10 \mathrm{eV}$, but the effect of Coulomb collisions becomes important at lower energies. Over the energy range of 30-55 eV (chosen to reduce the influence of the uncertainty in spacecraft charging), we find that the condition of local energy degradation is very well satisfied for dayside photoelectrons from 160 to $250 \mathrm{~km}$. No evidence of photoelectron transport is present over this energy range.
\end{abstract}

Keywords: Mars; Photoelectron; MAVEN

Citation: Wu, X. S., Cui, J., Yu, J., Liu, L. J., and Zhou, Z. J. (2019). Photoelectron balance in the dayside Martian upper atmosphere. Earth Planet. Phys., 3(5), 373-379. http://doi.org/10.26464/epp2019038

\section{Introduction}

Photoelectrons are an important population of non-thermal particles in the upper atmospheres of Solar System planets such as Earth (Doering et al., 1976; Lee et al., 1980a, b), Mars (Shutte et al., 1989; Frahm et al., 2006a, b; Sakai et al., 2015), Venus (Coates et al., 2008, 2015; Tsang et al., 2015), and Titan (Coates et al., 2007; Wellbrock et al., 2012). They are produced by solar Extreme Ultraviolet (EUV) and X-ray ionization of atmospheric neutrals, usually denoted as primary ionization, as well as by impact ionization from primary photoelectrons with energy above the local ionization potential, usually denoted as secondary ionization (Fox et al., 2008, and references therein). Photoelectrons are of extensive aeronomical interest because they represent a key link between solar EUV/X-ray energy and atmospheric thermal energy (e.g. Chen RH et al., 1978; Choi et al., 1998; Matta et al., 2014).

On Mars, the typical photoelectron energy distribution is characterized by sharp peaks at $22-27 \mathrm{eV}$ due to $\mathrm{CO}_{2}$ and $\mathrm{O}$ photoionization by the strong solar He II emission line at $30.4 \mathrm{~nm}$ (e.g. Frahm et al., 2006a, b). These peaks are superimposed on a smooth and continuous background up to an apparent knee at $60-70 \mathrm{eV}$ asso-

Correspondence to: J. Cui, cuijun7@mail.sysu.edu.cn

Received 04 JUN 2019; Accepted 30 JUN 2019.

Accepted article online 03 JUL 2019.

(C)2019 by Earth and Planetary Physics. ciated with the rapid drop in solar irradiance at wavelengths shorter than $17 \mathrm{~nm}$ (e.g. Sakai et al., 2015; Peterson et al., 2016). With increasing energy, another less prominent peak structure occurs near $500 \mathrm{eV}$ associated with inner shell Auger electrons due to X-ray ionization (e.g. Mitchell et al., 2000). Up to now, in situ measurements of photoelectrons have been made on various spacecrafts, such as the Mars Global Surveyor (e.g. Mitchell et al., 2000; Trantham et al., 2011; Liemohn et al., 2012; Xu SS et al., 2016a), the Mars Express (e.g. Frahm et al., 2006a, b, 2010; Han X et al., 2014), and the Mars Atmosphere and Volatile Evolution (MAVEN) (e.g. Sakai et al., 2015; Peterson et al., 2016; Xu et al., 2016b, 2017a, b), among others.

Upon production via either primary or secondary ionization, photoelectrons in the Martian upper atmosphere are subject to a number of collision processes including inelastic collisions with atmospheric neutrals leading to excitation or ionization (e.g. Bhardwaj and Jain, 2009), and Coulomb collisions with ionospheric thermal electrons (e.g. Stamnes and Rees, 1983). Assuming steady state, and at altitudes where the effect of transport is negligible, photoelectron production and loss should be in balance for any given energy and direction, a situation frequently referred to as local energy degradation (e.g. Mantas and Hanson, 1979).

This study is devoted to a verification of local energy degradation of photoelectrons observed in the Martian upper atmosphere. For 
such a purpose, information regarding a number of controlling factors is necessary, such as the solar EUV/X-ray flux, the photoelectron intensity, the neutral and electron densities, as well as the electron temperature. Simultaneous measurements of the above parameters became available with the arrival of MAVEN at Mars on 21 September 2014 (Jakosky et al., 2015).

This paper is organized as follows. We describe in Section 2 the formulism used to calculate various photoelectron production and loss rates. In Section 3 we present the main results from our detailed calculations based on a multi-instrument data set accumulated over a representative dayside MAVEN orbit. Finally, conclusions are summarized in Section 4.

\section{Formulism}

The various photoelectron production and loss processes considered in this study include primary and secondary ionization (Nicholson et al., 2009), inelastic collisions with atmospheric neutrals (Bhardwaj and Jain, 2009), and Coulomb collisions with ionospheric thermal electrons (Stamnes and Rees, 1983).

Following Lavvas et al. (2011), the primary production rate, $P_{\mathrm{e}}^{(\mathrm{ine})}$, is obtained from

$$
P_{\mathrm{e}}^{(\text {pri })}(z, E)=\frac{1}{4 \pi} \sum_{k, j} F_{\infty}(\lambda) \mathrm{e}^{-\tau(z, \theta, \lambda)} \sigma_{k, j}^{(\text {pi })}(\lambda) N_{k}(z),
$$

where $z$ is the altitude, $E$ is the photoelectron energy, $\lambda$ is the wavelength, $F_{\infty}$ is the solar flux at the top of the atmosphere, $\sigma_{k, j}^{(\mathrm{pi})}$ is the photoionization cross section for neutral species, $k$, and ionization channel, $j, N_{k}$ is the neutral density, and $\tau$ is the optical depth along the line-of-sight with $\theta$ being the solar zenith angle (SZA). For a given species and a given channel, the energy of the released photoelectron is $E=h c / \lambda-E_{k, j}^{(\text {th) }}$ where $h$ is the Planck constant, $c$ is the speed of light, and $E_{k, j}^{(\mathrm{th})}$ is the respective ionization potential. Similarly, the secondary ionization rate, $P_{\mathrm{e}}^{\mathrm{sec}}$, is obtained with

$$
P_{\mathrm{e}}^{(\mathrm{sec})}(z, E)=\sum_{k, j} \Phi_{\mathrm{e}}\left(z, E^{\prime}\right) \sigma_{k, j}^{(\mathrm{ei})}\left(E^{\prime}\right) N_{k}(z)
$$

where $\Phi_{\mathrm{e}}$ is the mean differential photoelectron intensity, $\sigma_{k, j}^{(\mathrm{ei})}$ is the electron impact ionization cross section, and the energy of the released secondary photoelectron, $E$, is connected to the energy of the impacting primary photoelectron, $E^{\prime}$, via $E=E^{\prime}-E_{k, j}^{\text {(th) }}$.

Collisions with atmospheric neutrals cause both production and loss of photoelectrons. The respective rates, denoted as $P_{\mathrm{e}}^{(\mathrm{ine})}$ and $L_{\mathrm{e}}^{\text {(ine) }}$, are given by

$$
P_{\mathrm{e}}^{\text {(ine) }}(z, E)=\sum_{k, j} \int_{E}^{\infty} \Phi_{\mathrm{e}}\left(z, E^{\prime}\right) R_{k, j}\left(E^{\prime}, E\right) N_{k}(z) \mathrm{d} E^{\prime},
$$

and

$$
L_{\mathrm{e}}^{(\text {ine) }}(z, E)=\sum_{k, j} \Phi_{\mathrm{e}}(z, E) \sigma_{k, j}^{(\mathrm{ine})}(E) N_{k}(z)
$$

where $R_{k, j}$ is the redistribution function and $\sigma_{k, j}^{(\text {ine) }}$ is the inelastic collision cross section for electron impact. The former reflects the probability that a photoelectron with energy $E^{\prime}$ is degraded to energy $E$ via various channels of collisional relaxation.
The photoelectron loss rate via Coulomb collisions, $L_{\mathrm{e}}^{(\mathrm{cmb})}$, can be calculated from

$$
L_{\mathrm{e}}^{(\mathrm{cmb})}(z, E)=-N_{\mathrm{e}}(z) \frac{\partial\left[S(z, E) \Phi_{\mathrm{e}}(z, E)\right]}{\partial E}
$$

with $N_{\mathrm{e}}$ being the thermal electron density and $S$ being the stopping cross section formulated as

$$
S(z, E)=\frac{3.37 \times 10^{-12}}{E^{0.94}\left[N_{\mathrm{e}}(z)\right]^{0.03}}\left[\frac{E-E_{\mathrm{e}}(z)}{E-0.53 E_{\mathrm{e}}(z)}\right]^{2.36}
$$

in unit of $\mathrm{eV} \cdot \mathrm{cm}^{2}$ following Stamnes and Rees (1983). In equation (6), $E_{\mathrm{e}}=8.618 \times 10^{-5} T_{\mathrm{e}}$ with $T_{\mathrm{e}}$ being the electron temperature in $\mathrm{K}$.

The formulism outlined above is implemented in this study assuming that all relevant parameters are isotropic (Lavvas et al., 2011). The redistribution function is parameterized similar to Lavvas et al. (2011). All necessary photon and electron impact cross sections are adapted from our previous compilation in Cui J et al. (2011).

\section{Photoelectron Balance During MAVEN Orbit No. 2909}

In this section, we verify the condition of local energy degradation for photoelectrons with the aid of the multi-instrument MAVEN data set, taking orbit No. 2909 on 28 March 2016 as an example. The periapsis altitude of this orbit is $158 \mathrm{~km}$ at a SZA of $67^{\circ}$. The $10.7 \mathrm{~cm}$ solar radio index at Earth during the period is $87 \mathrm{in}$ solar flux unit $\left(10^{-22} \mathrm{~W} \cdot \mathrm{m}^{-2}\right)$, indicating low solar activity conditions. The sampled regions are within the northern hemisphere of the planet characterized by draped magnetic field lines (e.g. Brain et al., 2006).

We consider a background neutral atmosphere composed of $\mathrm{CO}_{2}$, $\mathrm{O}, \mathrm{N}_{2}$, and $\mathrm{CO}$, with their densities extracted from the MAVEN Neutral Gas and lon Mass Spectrometer (NGIMS) measurements in the closed neutral source mode (Mahaffy et al., 2015). The thermal electron density is assumed to be equal to the total ion density based on the NGIMS measurements in the open source ion mode (Benna et al., 2015), and the electron temperature is based on the characteristics of the current voltage relation obtained by the MAVEN Langmuir Probe and Waves (LPW) (Ergun et al., 2015). The incident solar EUV/X-ray flux is adapted from the solar spectral model at Mars constructed from the Flare Irradiance Spectral Model-Mars and calibrated with the MAVEN Ultraviolet Monitor band irradiance data (Thiemann et al., 2017). Finally, the photoelectron energy distribution is available from the mean differential intensities of energetic electrons measured by the MAVEN Solar Wind Electron Analyzer (SWEA) (Mitchell et al., 2016). Each SWEA spectrum is corrected for spacecraft charging using the potential derived from MAVEN Suprathermal and Thermal Ion Composition measurements (McFadden et al., 2015).

An example of our calculations of the photoelectron production and loss rates is presented in Figures 1 and 2. In Figure 1, we show from left to right the variations of neutral densities (panel a), ion and electron densities (panel b), neutral and electron temperatures (panel c), spacecraft charging (panel d), and energetic electron distribution (panel e) along the MAVEN trajectory. Only the inbound portion of the orbit is included to reduce possible wall 

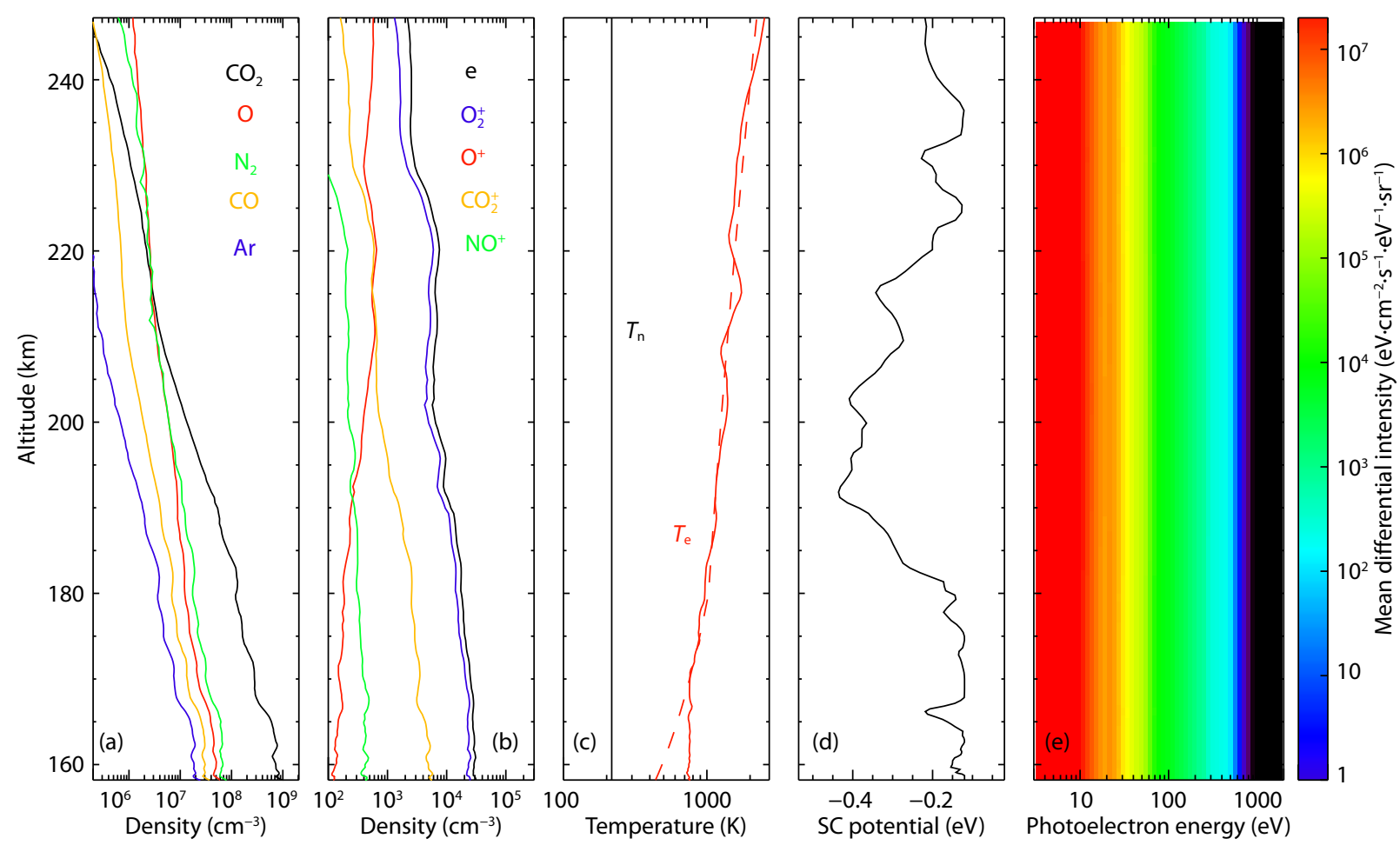

Figure 1. The variations of neutral densities (a), ion and electron densities (b), neutral and electron temperatures (c), spacecraft charging (d), and photoelectron energy distribution (e) along the inbound portion of MAVEN orbit No. 2909 on 28 March 2016, combining the multi-instrument data set acquired by several relevant MAVEN instruments (see text for details). The electron density is assumed to be identical to the total ion density. The neutral temperature profile is estimated from the isothermal fitting to the Ar density data; the electron temperature is corrected for the known effect of surface resistance or capacitance on the Langmuir Probe and Waves instrument sensor. The measured and corrected electron temperature profiles are distinguished as the solid and dashed lines in red in panel c.

contamination on the NGIMS antechamber walls, a well-known effect that is known to occur on other mass spectrometers with a similar design (Cui J et al., 2009). The electron temperatures measured by the LPW are known to be overestimated due to surface resistance or capacitance on the instrument sensor (Ergun et al., 2015), as indicated by the fact that the electron temperature at low altitudes is substantially higher than the neutral temperature but thermal coupling between all species is expected there (e.g. Matta et al., 2014, Peterson et al., 2018). Here the constant neutral temperature given by the solid line in black in panel $c$ is derived from the isothermal fitting to the atmospheric Ar density profile (Stone et al., 2018). Similar to Cui et al. (2019), we adopt an empirical method to correct for the LPW measurements of electron temperature below $180 \mathrm{~km}$. For comparison, the measured and corrected electron temperature profiles are indicated by the solid and dashed lines in red in panel c. The distinctive photoelectron characteristics, such as the peak structure at 22-27 eV, the knee at 60-70 eV, and another peak structure at $500 \mathrm{eV}$, are clearly seen in the photoelectron energy spectrogram in panel e.

Based on the MAVEN measurements presented in Figure 1, equations (1)-(6) are used to calculate various photoelectron production and loss rates, which are displayed in Figure 2 including production via primary (panel a) and secondary (panel b) ionization, production (panel c) and loss (panel d) via inelastic collisions with atmospheric neutrals, and loss via Coulomb collisions with ionospheric thermal electrons (panel e). For a closer look, we compare in Figure 3 the energy distributions of these rates at two representative altitudes: $160 \mathrm{~km}$ near the periapsis (panel a) and $200 \mathrm{~km}$ (panel b). Both panels suggest that the contribution of secondary ionization is of minor importance, consistent with the relatively small ionization efficiency reported by Cui J et al. (2018) also based on the MAVEN data. For the two photoelectron loss processes, the relative importance of Coulomb collisions clearly increases with decreasing photoelectron energy. Meanwhile, it is more prominent at higher altitudes, likely connected to an increasing thermal electron to neutral density ratio which is $3.7 \times 10^{-5}$ at $160 \mathrm{~km}$ and $3.3 \times 10^{-4}$ at $200 \mathrm{~km}$, respectively. According to Figures 2 and 3, the photoelectron balance is controlled mainly by primary production, as well as by production and loss via inelastic collisions with atmospheric neutrals, over the energy range considered here (see below).

The calculated photoelectron production and loss rates are subject to several sources of uncertainty. The typical uncertainty is $10 \%$ in solar flux according to Thiemann et al. (2017) and $10 \%$ in photoionization cross section according to Heays et al. (2017), implying a combined uncertainty of $14 \%$ in primary ionization where the small uncertainty of less than $1 \%$ in neutral density is ignored. The uncertainty in differential photoelectron intensity is about $10 \%$ (Mitchell et al., 2016) and the uncertainty in electron impact cross section is about $5 \%$ (Itikawa, 2002). These lead to a combined uncertainty of $11 \%$ in secondary ionization. Following the same line of reasoning, we estimate the uncertainties in photo- 
(a) $P_{\mathrm{e}}^{\text {(pri) }}$

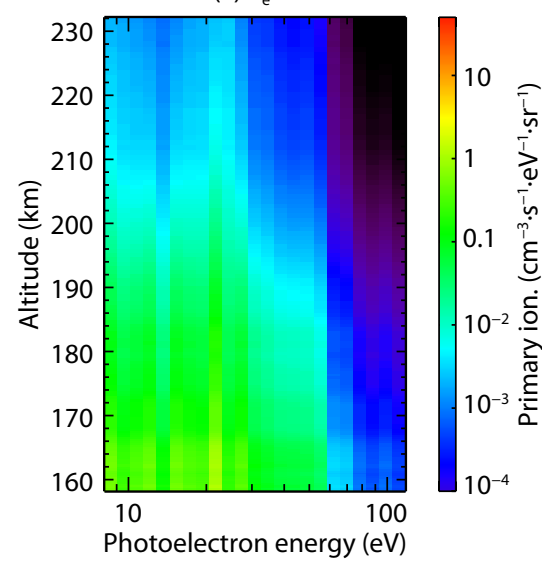

(b) $P_{\mathrm{e}}^{\text {(sec) }}$

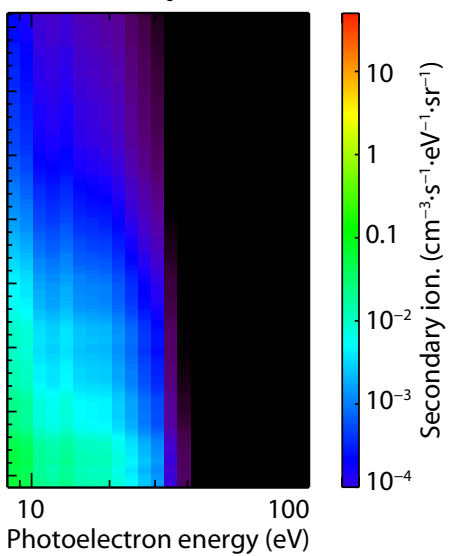

(e) $L_{e}^{(\mathrm{cmb})}$

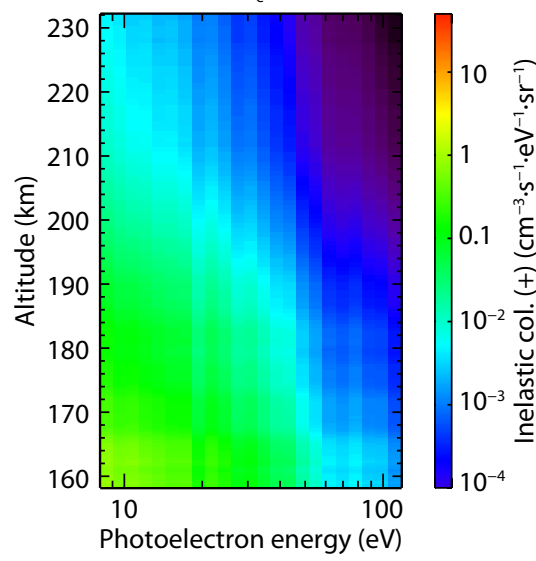

(d) $L_{\mathrm{e}}^{\text {(ine) }}$

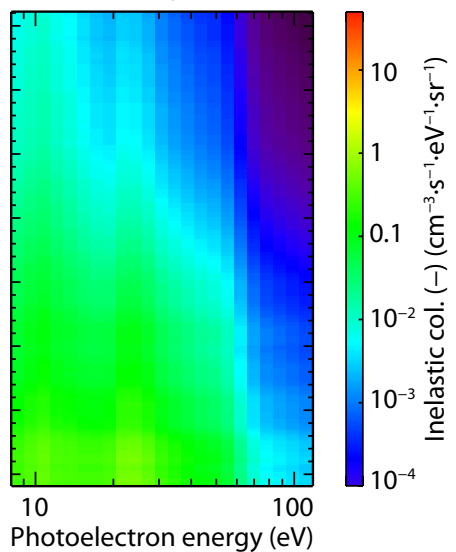

Figure 2. The full altitude variations of different photoelectron production and loss rates along the inbound portion of MAVEN orbit No. 2909, including production via primary (a) and secondary (b) ionization, production (c) and loss (d) via inelastic collisions with atmospheric neutrals, as well as loss via Coulomb collisions with ionospheric thermal electrons (e).

(a) $160 \mathrm{~km}$

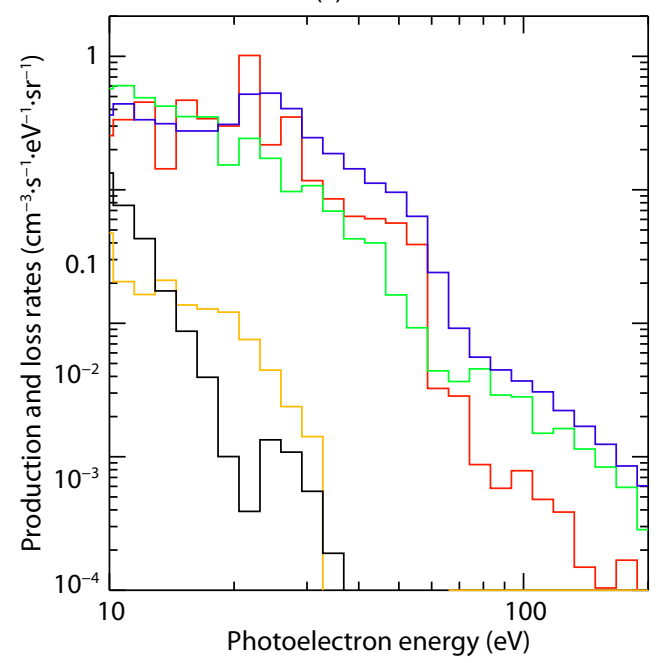

(b) $200 \mathrm{~km}$

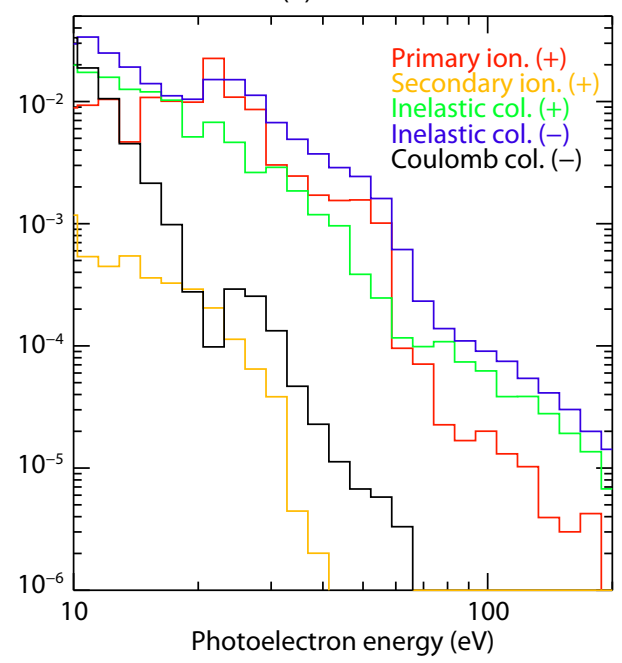

Figure 3. The energy distributions of the photoelectron production (indicated by " + ") and loss (indicated by "-") rates at two representative altitudes of $160 \mathrm{~km}$ near the periapsis (a) and $200 \mathrm{~km}$ (b), both over the inbound portion of MAVEN orbit No. 2909. Different colors represent different processes including primary and secondary ionization, production and loss via inelastic collisions with atmospheric neutrals, as well as Coulomb collisions with ionospheric thermal electrons. 


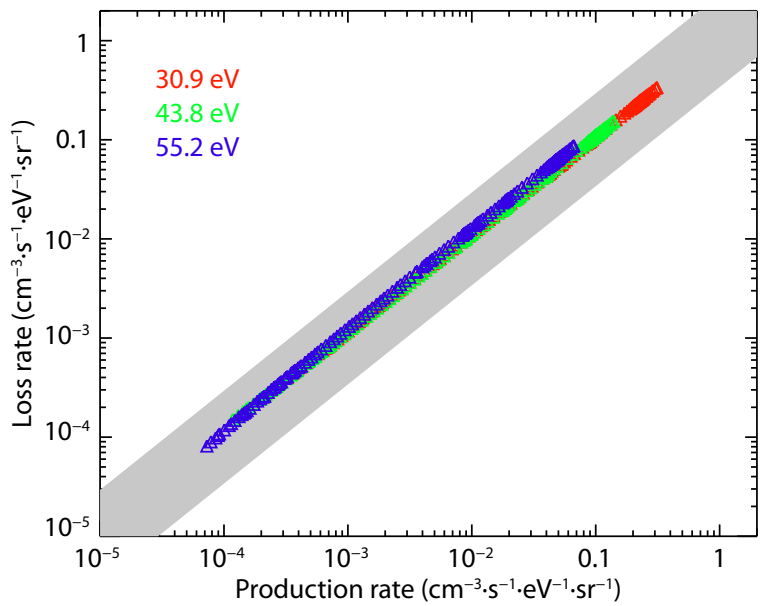

Figure 4. The relation between photoelectron production and loss rates over the inbound portion of MAVEN orbit No. 2909. Different colors stand for different photoelectron energies, as indicated in the figure legend. The region encompassing the combined measurement uncertainty is shadowed for reference.

electron production and in loss via inelastic collisions both to be $11 \%$ as well. The uncertainty in loss via Coulomb collisions is primarily determined by the uncertainty in thermal electron density, which is $25 \%$ according to Benna et al. (2015). The uncertainty in electron temperature, though as large as $20 \%$ according to Ergun et al. (2015), is not important here due to the insensitivity of the stopping function to this parameter (see equation (6)). From all the above, we conclude that the uncertainties in total production and loss to be $21 \%$ and $27 \%$, respectively. However, we caution that a small uncertainty in spacecraft charging would lead to large uncertainties in the calculated production and loss rates at energies where an inherent rapid change in differential photoelectron intensity is expected, i.e. near the peaks at 22-27 eV and near the knee at $60-70 \mathrm{eV}$. To avoid such an undesired situation, we evaluate below the condition of local energy degradation over a restricted energy range - from 30 to $55 \mathrm{eV}$ only.

Figure 2 reveals that, at $160 \mathrm{~km}$, the total photoelectron production rate, which is the sum of $P_{\mathrm{e}}^{\mathrm{pri}}, P_{\mathrm{e}}^{\mathrm{sec}}$, and $P_{\mathrm{e}}^{\mathrm{ine}}$, is generally consistent with the total loss rate, which is the sum of $L_{e}^{\text {ine }}$ and $L_{e}^{\mathrm{cmb}}$, with a median difference of $17 \%$ for any given energy between 30-55 eV. At $200 \mathrm{~km}$, the agreement between production and loss is improved, with a median difference of just $13 \%$. Both are well below the combined measurement uncertainties quoted above, implying that the condition of local energy degradation is satisfied. A more detailed comparison between the photoelectron production and loss rates is presented in Figure 4 over the altitude range of 160 to $250 \mathrm{~km}$ for several arbitrarily chosen energies (of $30.9 \mathrm{eV}, 43.8 \mathrm{eV}$, and $55.2 \mathrm{eV}$, respectively) where the region encompassing the combined measurement uncertainty is shadowed for reference. The figure clearly indicates that local energy degradation is a very good approximation in the dayside Martian ionosphere at altitudes considered here.

Interestingly, the analysis presented here reveals no evidence for photoelectron transport over the considered energy range, implying that either transport occurs within the more tenuous part of the Martian upper atmosphere (e.g. Cui J et al., 2011) or alternatively a large potential drop is present in the distant regions to reflect photoelectrons escaping back towards the planet (e.g. Kitamura et al., 2015). A thorough investigation of such an issue clearly relies on the evaluation of local energy degradation over the full energy range measured by the SWEA, which is left for follow-up studies.

\section{Concluding Remarks}

Photoelectrons are created by solar EUV/X-ray ionization and serve as an important link between solar radiative energy and atmospheric thermal energy (Fox et al., 2008, and references therein). Observations have been made of photoelectrons near many Solar System objects such as Earth, Mars, Venus, and Titan (Coates et al., 2011). At altitudes where transport is negligible, the balance of photoelectrons is controlled by local energy degradation, a condition for which production is strictly balanced by loss (e.g. Lavvas et al., 2011).

In this study, we attempt to verify the condition of local energy degradation with the aid of the combined MAVEN measurements that provide a complete set of the physical parameters required for calculating various photoelectron production and loss rates. Taking MAVEN orbit No. 2909 as an example, our calculations indicate that photoelectron production occurs mainly via primary ionization as well as degradation from higher energy states via inelastic collisions with ambient neutrals, and photoelectron loss occurs mainly via degradation towards lower energy states also via inelastic collisions, but the effect of Coulomb collisions with ambient thermal electrons becomes important for photoelectrons less energetic than $10 \mathrm{eV}$. The condition of local energy degradation is evaluated over the energy range of 30-55 eV, chosen to reduce the influence of the uncertainty in spacecraft charging. Our analysis reveals that such a condition is very well satisfied for dayside photoelectrons up to at least $230 \mathrm{~km}$. It will be interesting to examine in follow-up studies the questions of at what altitudes and what energies the condition of local energy degradation is broken and transport becomes important.

\section{Acknowledgments}

J Cui acknowledges supports from the National Natural Science Foundation of China (NNSFC) through grants 41525015 and 41774186. The data used in this study are publicly available at the MAVEN Science Data Center (https://lasp.colorado.edu/maven/ sdc/public/).

\section{References}

Benna, M., Mahaffy, P. R., Grebowsky, J. M., Fox, J. L., Yelle, R. V., and Jakosky, B. M. (2015). First measurements of composition and dynamics of the Martian ionosphere by MAVEN's Neutral Gas and Ion Mass Spectrometer. Geophys. Res. Lett., 42(21), 8958-8965. https://doi.org/10.1002/2015GL066146

Bhardwaj, A., and Jain, S. K. (2009). Monte Carlo model of electron energy degradation in a $\mathrm{CO}_{2}$ atmosphere. J. Geophys. Res. Space Phys., 114(A11), A11309. https://doi.org/10.1029/2009JA014298

Brain, D. A., Mitchell, D. L., and Halekas, J. S. (2006). The magnetic field draping direction at Mars from April 1999 through August 2004. Icarus, 182(2), 464-473. https://doi.org/10.1016/j.icarus.2005.09.023

Chen, R. H., Cravens, T. E., and Nagy, A. F. (1978). The Martian ionosphere in light 
of the Viking observations. J. Geophys. Res. Space Phys., 83(A8), 3871-3876. https://doi.org/10.1029/JA083iA08p03871

Choi, Y. W., Kim, J., Min, K. W., Nagy, A. F., and Oyama, K. I. (1998). Effect of the magnetic field on the energetics of Mars ionosphere. Geophys. Res. Lett., 25(14), 2753-2756. https://doi.org/10.1029/98GL51839

Coates, A. J., Frahm, R. A., Linder, D. R., Kataria, D. O., Soobiah, Y., Collinson, G., Sharber, J. R., Winningham, J. D., Jeffers, S. J., ... Grande, M. (2008). Ionospheric photoelectrons at Venus: Initial observations by ASPERA-4 ELS. Planet. Space Sci, 56(6), 802-806. https://doi.org/10.1016/j.pss.2007.12.008

Coates, A. J., Crary, F. J., Young, D. T., Szego, K., Arridge, C. S., Bebesi, Z., Sittler, Jr. E. C., Hartle, R. E., and Hill, T. W. (2007). Ionospheric electrons in Titan's tail: Plasma structure during the Cassini T9 encounter. Geophys. Res. Lett., 34(24), L24S05. https://doi.org/10.1029/2007GL030919

Coates, A. J., Tsang, S. M. E., Wellbrock, A., Frahm, R. A., Winningham, J. D., Barabash, S., Lundin, R., Young, D. T., and Crary, F. J. (2011). Ionospheric photoelectrons: Comparing Venus, Earth, Mars and Titan. Planet. Space Sci., 59(10), 1019-1027. https://doi.org/10.1016/j.pss.2010.07.016

Coates, A. J., Wellbrock, A., Frahm, R. A., Winningham, J. D., Fedorov, A., Barabash, S., and Lundin, R. (2015). Distant ionospheric photoelectron energy peak observations at Venus. Planet. Space Sci., 113-114, 378-384. https://doi.org/10.1016/j.pss.2015.02.003

Cui, J., Yelle, R. V., Vuitton, V., Waite, Jr. J. H., Kasprzak, W. T., Gell, D. A., Niemann, H. B., Müller-Wodarg, I. C. F., Borggren, N., ... Magee, B. A. (2009). Analysis of Titan's neutral upper atmosphere from Cassini lon Neutral Mass Spectrometer measurements. Icarus, 200(2), 581-615. https://doi.org/10.1016/j.icarus.2008.12.005

Cui, J., Galand, M., Coates, A. J., Zhang, T. L., and Müller-Wodarg, I. C. F. (2011). Suprathermal electron spectra in the Venus ionosphere. J. Geophys. Res. Space Phys., 116(A4), A04321. https://doi.org/10.1029/2010JA016153

Cui, J., Wu, X. S., Xu, S. S., Wang, X. D., Wellbrock, A., Nordheim, T. A., Cao, Y. T., Wang, W. R., Sun, W. Q., ... Wei, Y. (2018). lonization efficiency in the dayside Martian upper atmosphere. Astrophys. J. Lett., 857(2), L18. https://doi.org/10.3847/2041-8213/aabcc6

Cui, J., Cao, Y. T., Wu, X. S., Xu, S. S., Yelle, R. V., Stone, S., Vigren, E., Edberg, N. J. T., Shen, C. L., He, F., and Wei, Y. (2019). Evaluating local ionization balance in the nightside Martian upper atmosphere during MAVEN Deep Dip campaigns. Astrophys. J. Lett., 876(1), L12. https://doi.org/10.3847/20418213/ab1b34

Doering, J. P., Peterson, W. K., Bostrom, C. O., and Potemra, T. A. (1976). High resolution daytime photoelectron energy spectra from AE-E. Geophys. Res. Lett., 3(3), 129-131. https://doi.org/10.1029/GL003i003p00129

Ergun, R. E., Morooka, M. W., Andersson, L. A., Fowler, C. M., Delory, G. T., Andrews, D. J., Eriksson, A. I., McEnulty, T., and Jakosky, B. M. (2015). Dayside electron temperature and density profiles at Mars: First results from the MAVEN Langmuir probe and waves instrument. Geophys. Res. Lett., 42(21), 8846-8853. https://doi.org/10.1002/2015GL065280

Fox, J. L., Galand, M. I., and Johnson, R. E. (2008). Energy deposition in planetary atmospheres by charged particles and solar photons. Space Sci. Rev., 139(14), 3-62. https://doi.org/10.1007/s11214-008-9403-7

Frahm, R. A., Winningham, J. D., Sharber, J. R., Scherrer, J. R., Jeffers, S. J., Coates, A. J., Linder, D. R., Kataria, D. O., Lundin, R., ... Dierker, C. (2006a). Carbon dioxide photoelectron energy peaks at Mars. Icarus, 182(2), 371-382. https://doi.org/10.1016/j.icarus.2006.01.014

Frahm, R. A., Sharber, J. R., Winningham, J. D., Wurz, P., Liemohn, M. W., Kallio, E., Yamauchi, M., Lundin, R., Barabash, S., ... McKenna-Lawer, S. (2006b). Locations of atmospheric photoelectron energy peaks within the Mars environment. Space Sci. Rev., 126(1-4), 389-402. https://doi.org/10.1007/s11214-006-9119-5

Frahm, R. A., Sharber, J. R., Winningham, J. D., Link, R., Liemohn, M. W., Kozyra, J. U., Coates, A. J., Linder, D. R., Barabash, S., ... Fedorov, A. (2010). Estimation of the escape of photoelectrons from Mars in 2004 liberated by the ionization of carbon dioxide and atomic oxygen. Icarus, 206(1), 50-63. https://doi.org/10.1016/j.icarus.2009.03.024

Han, X., Fraenz, M., Dubinin, E., Wei, Y., Andrews, D. J., Wan, W., He, M., Rong, Z. J., Chai, L., ... Barabash, S. (2014). Discrepancy between ionopause and photoelectron boundary determined from Mars Express measurements. Geophys. Res. Lett., 41(23), 8221-8227.

https://doi.org/10.1002/2014GL062287

Heays, A. N., Bosman, A. D., and van Dishoeck, E. F. (2017). Photodissociation and photoionisation of atoms and molecules of astrophysical interest. Astron. Astrophys., 602, A105. https://doi.org/10.1051/0004-6361/201628742

Itikawa, Y. (2002). Cross sections for electron collisions with carbon dioxide. J. Phys. Chem. Ref. Data, 31(3), 749-769. https://doi.org/10.1063/1.1481879

Jakosky, B. M., Grebowsky, J. M., Luhmann, J. G., and Brain, D. A. (2015). Initial results from the MAVEN mission to Mars. Geophys. Res. Lett., 42(21), 8791-8802. https://doi.org/10.1002/2015GL065271

Kitamura, N., Seki, K., Nishimura, Y., and McFadden, J. P. (2015). Limited impact of escaping photoelectrons on the terrestrial polar wind flux in the polar cap. Geophys. Res. Lett., 42(9), 3106-3113. https://doi.org/10.1002/2015GL063452

Lavvas, P., Galand, M., Yelle, R. V., Heays, A. N., Lewis, B. R., Lewis, G. R., and Coates, A. J. (2011). Energy deposition and primary chemical products in Titan's upper atmosphere. Icarus, 213(1), 233-251. https://doi.org/10.1016/j.icarus.2011.03.001

Lee, J. S., Doering, J. P., Potemra, T. A., and Brace, L. H. (1980). Measurements of the ambient photoelectron spectrum from atmosphere explorer: I . AE-E measurements below $300 \mathrm{~km}$ during solar minimum conditions. Planet. Space Sci., 28(10), 947-971. https://doi.org/10.1016/0032-0633(80)90058-6

Lee, J. S., Doering, J. P., Potemra, T. A., and Brace, L. H. (1980). Measurements of the ambient photoelectron spectrum from atmosphere explorer: II. AE-E measurements from 300 to $1000 \mathrm{~km}$ during solar minimum conditions. Planet. Space Sci., 28(10), 973-996. https://doi.org/10.1016/00320633(80)90059-8

Liemohn, M. W., Dupre, A., Bougher, S. W., Trantham, M., Mitchell, D. L., and Smith, M. D. (2012). Time-history influence of global dust storms on the upper atmosphere at Mars. Geophys. Res. Lett., 39(11), L11201. https://doi.org/10.1029/2012GL051994

Mahaffy, P. R., Benna, M., Elrod, M., Yelle, R. V., Bougher, S. W., Stone, S. W., and Jakosky, B. M. (2015). Structure and composition of the neutral upper atmosphere of Mars from the MAVEN NGIMS investigation. Geophys. Res. Lett., 42(21), 8951-8957. https://doi.org/10.1002/2015GL065329

Mantas, G. P., and Hanson, W. B. (1979). Photoelectron fluxes in the Martian ionosphere. J. Geophys. Res., 84(A2), 369-385. https://doi.org/10.1029/JA084iA02p00369

Matta, M., Galand, M., Moore, L., Mendillo, M., and Withers, P. (2014). Numerical simulations of ion and electron temperatures in the ionosphere of Mars: Multiple ions and diurnal variations. Icarus, 227, 78-88. https://doi.org/10.1016/j.icarus.2013.09.006

McFadden, J. P., Kortmann, O., Curtis, D., Dalton, G., Johnson, G., Abiad, R., Sterling, R., Hatch, K., Berg, P., ... Jakosky, B. (2015). MAVEN suprathermal and thermal ion compostion (STATIC) instrument. Space Sci. Rev., 195(1-4), 199-256. https://doi.org/10.1007/s11214-015-0175-6

Mitchell, D. L., Lin, R. P., Rème, H., Crider, D. H., Cloutier, P. A., Connerney, J. E. P., Acuña, M. H., and Ness, N. F. (2000). Oxygen auger electrons observed in Mars' ionosphere. Geophys. Res. Lett., 27(13), 1871-1874. https://doi.org/10.1029/1999GL010754

Mitchell, D. L., Mazelle, C., Sauvaud, J. A., Thocaven, J. J., Rouzaud, J., Fedorov, A., Rouger, P., Toublanc, D., Taylor, E., ... Jakosky, B. M. (2016). The MAVEN solar wind electron analyzer. Space Sci. Rev., 200(1-4), 495-528. https://doi.org/10.1007/s11214-015-0232-1

Nicholson, W. P., Gronoff, G., Lilensten, J., Aylward, A. D., and Simon, C. (2009). A fast computation of the secondary ion production in the ionosphere of Mars. Mon. Not. R. Astron. Soc., 400(1), 369-382.

https://doi.org/10.1111/j.1365-2966.2009.15463.x

Peterson, W. K., Thiemann, E. M. B., Eparvier, F. G., Andersson, L., Fowler, C. M., Larson, D., Mitchell, D., Mazelle, C., Fontenla, J., ... Jakosky, B. (2016). Photoelectrons and solar ionizing radiation at Mars: Predictions versus MAVEN observations. J. Geophys. Res. Space Phys., 121(9), 8859-8870. https://doi.org/10.1002/2016JA022677

Peterson, W. K., Fowler, C. M., Andersson, L. A., Thiemann, E. M. B., Jain, S. K., 
Mayyasi, M., Esman, T. M., Yelle, R., Benna, M., and Espley, J. (2018). Martian electron temperatures in the subsolar region: MAVEN observations compared to a one-dimensional model. J. Geophys. Res. Space Phys., 123(7), 5960-5973. https://doi.org/10.1029/2018JA025406

Sakai, S., Rahmati, A., Mitchell, D. L., Cravens, T. E., Bougher, S. W., Mazelle, C., Peterson, W. K., Eparvier, F. G., Fontenla, J. M., and Jakosky, B. M. (2015). Model insights into energetic photoelectrons measured at Mars by MAVEN. Geophys. Res. Lett., 42(21), 8894-8900. https://doi.org/10.1002/2015GL065169

Shutte, N. M., Király, P., Cravens, T. E., Dyachkov, A. V., Gombos, T. I., Gringuaz, K. I., Nagy, A. F., Sharp, W. E., Sheronova, S. M., ... Verigin, M. (1989). Observation of electron and ion fluxes in the vicinity of Mars with the HARP spectrometer. Nature, 341(6243), 614-616. https://doi.org/10.1038/341614a0

Stamnes, K., and Rees, M. H. (1983). Heating of thermal ionospheric electrons by suprathermal electrons. Geophys. Res. Lett., 10(4), 309-312. https://doi.org/10.1029/GL010i004p00309

Stone, S. W., Yelle, R. V., Benna, M., Elrod, M. K., and Mahaffy, P. R. (2018). Thermal structure of the Martian upper atmosphere from MAVEN NGIMS. J. Geophys. Res. Planets, 123(11), 2842-2867. https://doi.org/10.1029/2018JE005559

Thiemann, E. M. B., Chamberlin, P. C., Eparvier, F. G., Templeman, B., Woods, T. N., Bougher, S. W., and Jakosky, B. M. (2017). The MAVEN EUVM model of solar spectral irradiance variability at Mars: Algorithms and results. J. Geophys. Res. Space Phys., 122(3), 2748-2767. https://doi.org/10.1002/2016JA023512

Trantham, M., Liemohn, M., Mitchell, D., and Frank, J. (2011). Photoelectrons on closed crustal field lines at Mars. J. Geophys. Res. Space Phys., 116(A7),
A07311. https://doi.org/10.1029/2010JA016231

Tsang, S. M. E., Coates, A. J., Jones, G. H., Frahm, R. A., Winningham, J. D., Barabash, S., Lundin, R., and Fedorov, A. (2015). lonospheric photoelectrons at Venus: Case studies and first observation in the tail. Planet. Space Sci., 113114, 385-394. https://doi.org/10.1016/j.pss.2015.01.019

Wellbrock, A., Coates, A. J., Sillanpää, I., Jones, G. H., Arridge, C. S., Lewis, G. R., Young, D. T., Crary, F. J., and Aylward, A. D. (2012). Cassini observations of ionospheric photoelectrons at large distances from Titan: Implications for Titan's exospheric environment and magnetic tail. J. Geophys. Res. Space Phys., 117(A3), A03216. https://doi.org/10.1029/2011JA017113

Xu, S. S., Liemohn, M., Bougher, S., and Mitchell, D. (2016a). Martian highaltitude photoelectrons independent of solar zenith angle. J. Geophys. Res. Space Phys., 121(4), 3767-3780. https://doi.org/10.1002/2015JA022149

Xu, S. S., Mitchell, D., Liemohn, M., Dong, C. F., Bougher, S., Fillingim, F., Lillis, R., McFadden, J., Mazelle, C., ... Jakosky, B. (2016b). Deep nightside photoelectron observations by MAVEN SWEA: Implications for Martian northern hemispheric magnetic topology and nightside ionosphere source. Geophys. Res. Lett., 43(17), 8876-8884. https://doi.org/10.1002/2016GL070527

Xu, S. S., Mitchell, D., Liemohn, M., Fang, X. H., Ma, Y. J., Luhmann, J., Brain, D., Steckiewicz, M., Mazelle, C., ... Jakosky, B. (2017a). Martian low-altitude magnetic topology deduced from MAVEN/SWEA observations. J. Geophys. Res. Space Phys., 122(2), 1831-1852. https://doi.org/10.1002/2016JA023467

Xu, S. S., Mitchell, D., Luhmann, J., Ma, Y. J., Fang, X. H., Harada, Y., Hara, T., Brain, D., Weber, T., ... DiBraccio, G. A. (2017b). High-altitude closed magnetic loops at Mars observed by MAVEN. Geophys. Res. Lett., 44(22), 11229-11238. https://doi.org/10.1002/2017GL075831 